\title{
Efficacy of Primary Posterior Curvilinear Capsulorhexis in Adults
}

\author{
Dr. M.V.D.L Sathyanarayana M.S \\ Assistant Professor ofOphthalmology RI.M.S Kadapa A.PDR.B. Keerthi P.G R.I.M.S Kadapa A.P
}

\begin{abstract}
Posterior capsular opacification(pco) is the most frequent complication of cataract surgery anddevelops from residual lens epithelial cells that undergo proliferation,migration,metaplasia and differentiation in the capsular bag after cataract surgery.patients with PCO have decreased visual visual acuity, loss of contrast sensitivity, and in some cases, PCO precludes examination of the posterior segment. The incidence of PCO is reported to be 20-50\% 5 years after cataract surgery in the general population and has been reported to occur in 100\% of pediatric patients. Recently, primary posterior capsulorhexis (PPCC) has been proposed to prevent PCO in adults. The PPCC technique is frequently used in children with or without anterior vitrectomyto prevent posterior capsule opacification (PCO) but is rarely used in adults. In PPCC, the central portion of the posterior capsule is removed during cataract surgery to prevent equatorial LEC migration toward the visual axis.

Aim: The aim of the current study was to report the long-term efficacy and safety of phacoemulsification and manual phaco with posterior chamber intra ocular lens implantation combined with PPCC in adult patients with cataract.

Materials And Methods: In an interventional case series 100 patients underwent cataract surgery combined with PPCC at Rajiv Gandhi Institute of Medical Sciences Kadapa, Andhra Pradesh from August 2014 to June 2016. Informed consent was obtained from all patients preoperatively. Only adult patients who underwent cataract surgery and PPCC for age-related cataract were included the study.

Inclusion Criteria: Although we do not routinely perform PPCC during cataract surgery in adults, it is performed for cases with residual posterior capsule opacification despite careful polishing.

Excleusion Criteria: Patients with glaucoma, uveitis, zonular weakness, and proliferative diabetic retinopathy were excluded in this study. Patients with clear posterior capsule were also excluded.

Keywords:posterior capsular opacification, Nd: YAG capsulotomy, phacoemulsification, manualphaco, intra ocular lens, and primary posterior capsulorhexis
\end{abstract}

\section{Surgical Procedure}

All patients underwent preoperative examinations including measurement of the corrected distance visual acuity (CDVA) with a Snellen chart, slit-lamp bio microscopy, intraocular pressure (IOP) measurement using applanation tonometry, posterior segment visualization by indirect ophthalmoscopy and ultrasonography when the fundus could not be visualized. Patients were undergone either MANUAL PHACO orPhacoemulsification with posterior chamber intra ocular lens implantation either foldable or rigid lens. All patients underwent surgery with peribulbar block. Topical phenylephrine $2.5 \%$ and tropicamid $1 \%$ were used for preoperative pupil dilatation. After removing nucleus the capsular bag was filled with sodium hyaluronate $1 \%$, a flap was created using a 27 -gauge needle at the center of the posterior capsule. A small amount of sodium hyaluronate was injected through the capsular opening to separate the underlying anterior hyaloidphase from the posterior capsule. Then, the edge of the incised capsule was grasped with capsulorhexisforceps and the incision was extended peripherally to create a well-centred 3-4mm PPCC opening. Then intraocular lens with a $6 \mathrm{~mm}$ optic diameter was implanted inthe capsular bag. The sodium hyaluronate-chondroitin sulfate was aspirated from the anterior chamber and the incisions were self-sealing. Postoperatively, patients were instructed to instil topically ciprofloxacin and dexamethasone 6times a day. Demographic data including age, gender, number of eyes, and follow-up time were collected. Postoperatively, patients were assessed at 1 day, 1 week, 1 month, 3 months, 6 months, 1 year, and a final visit at various times. All statistical analyses were performed using SPSS for Windows, Version 16.0 (SPSS, Inc., Chicago, IL, USA). The paired T-test and Chi-square test were used to compare means with statistical significance at P0.05) [Figure 1]. At the last visit, $87.1 \%$ of the eyes had CDVA $\geq 20 / 30$ and $13 \%$ were $\geq 20 / 40$.

II. Results

\begin{tabular}{|l|l|c|}
\hline The mean age isTotal no. & No of males & No of females \\
\hline 100 & 55 & 45 \\
\hline
\end{tabular}




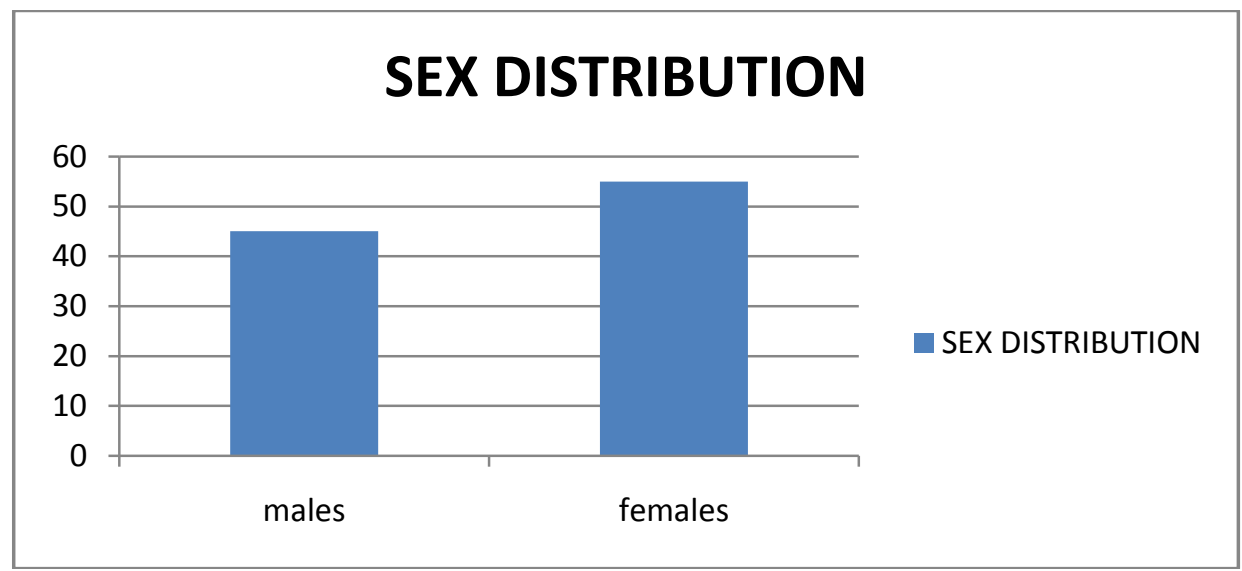

\begin{tabular}{|l|l|l|}
\hline Total no of eyes & Right eye & Left eye \\
\hline 100 & 61 & 39 \\
\hline
\end{tabular}

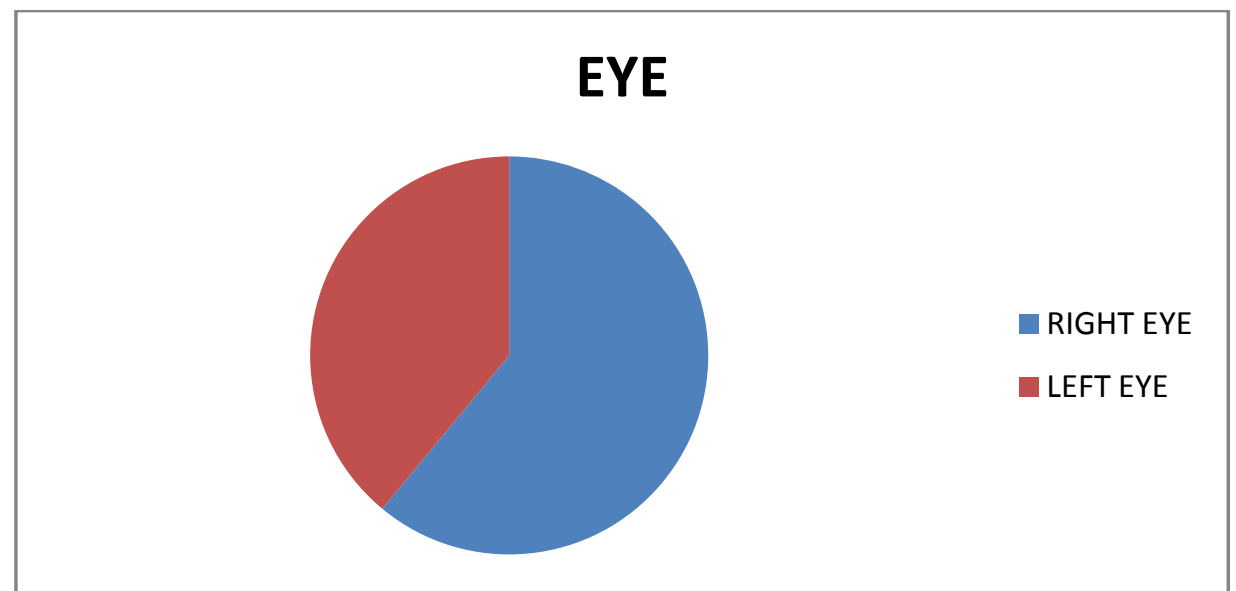

\section{Visual Outcome}

The mean preoperative BCVA was $0.26+-0.18$ Post-operative at $1^{\text {st }}$ week and final visit was $0.75+/-0.23$ and $0.78+/-0.24$.

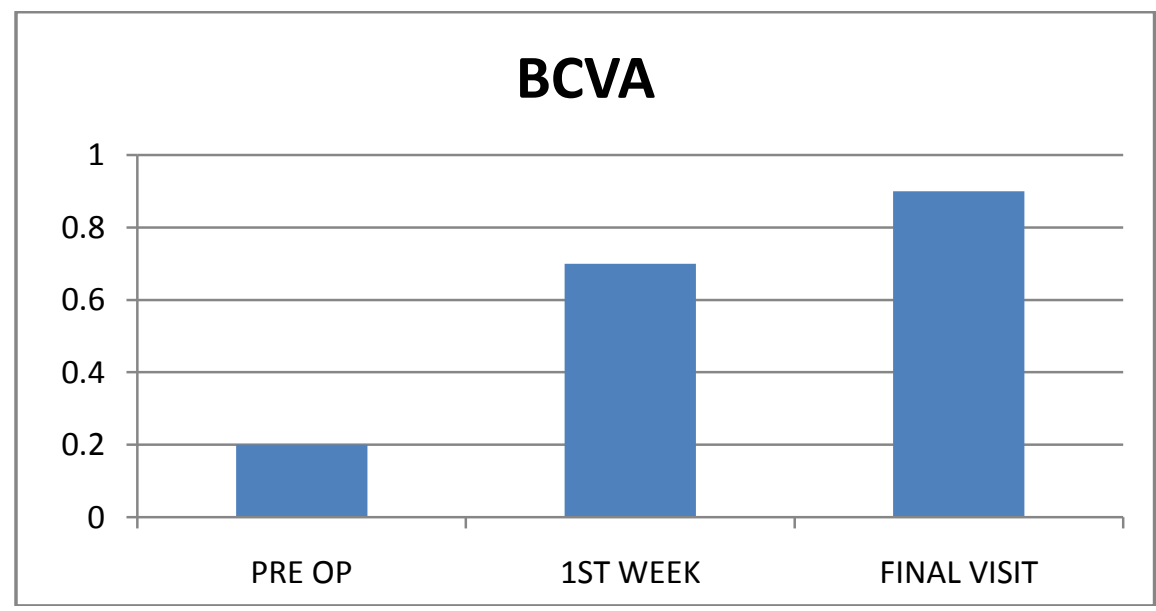

Perioperative and postoperative complications

\begin{tabular}{|l|l|}
\hline Retinal detachment & 0 \\
\hline Cme & 0 \\
\hline Peripheral extension & 2 \\
\hline Vitreous prolapse & 2 \\
\hline
\end{tabular}


No major intraoperative complicationsoccurred. In twocases rhexis went peripherally but completed. In another two cases vitreous prolapse happened and vitrectomy was done. Postoperatively, no serious complications, such as endophthalmitis or retinal detachment, were recorded.

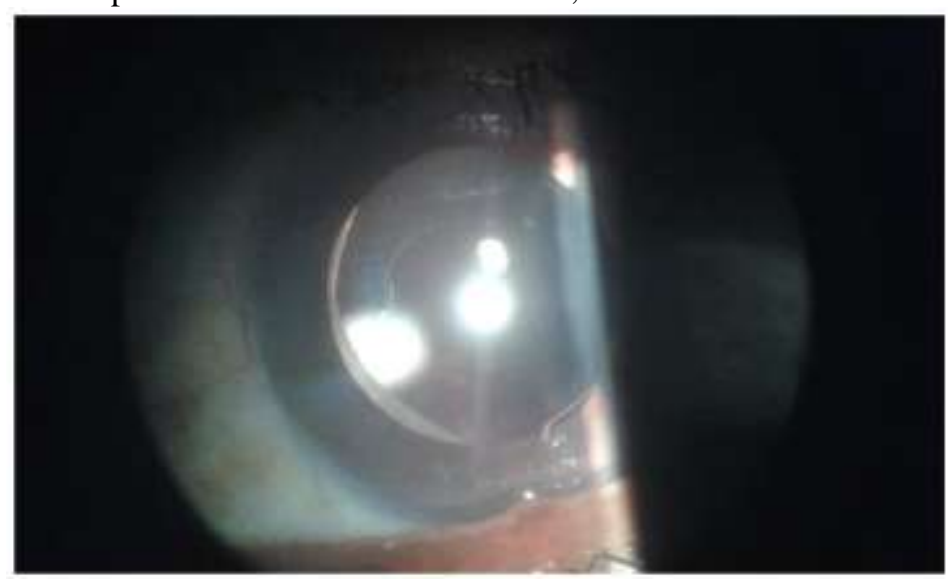

PHACOEMULSIFICATION WITH FOLDABLE IOL. AND PPCC

\section{Discussion}

- $\quad$ PCO referred to as 'secondary cataract' or 'after cataract', develops over the clear posterior capsule a few months to a few years after an uneventful cataract surgery. PCO results from the growth and abnormal proliferation of lens epithelial cells (LECs) on the capsule at the time of cataract surgery. These cells migrate to the posterior capsule, where they approach the central visual axis and cause visual-axis obscuration, resulting in dimness of vision. The PCO has two forms, fibrous and pearl. Sometimes, a combination of both is also found. Clinically, it is seen as a wrinkling on the posterior capsule at the site of fusion of the anterior and posterior capsules. A histological examination of the fibrous PCO shows extracellular matrix accumulation and the presence of elongated myofibroblast cells positive to vimentin and $\alpha$-smooth muscle actin.Examination of the proliferative PCO shows clusters of swollen, opacified, differentiated LECs called bladder or Wedlcells. The development of PCO is a very dynamic process and involves three basic phenomena: proliferation, migration and differentiation of residual LECs.

\section{Risk factors:}

- Nonmodifiable: Several systemic and ocular associations have been cited for influencing the development of PCO. Age remains a confounding factor in the entire process of development of posterior capsuleopacification, with younger individuals at a higher risk

\section{Modifiable Surgical Techniques}

- Continuous Curvilinear Capsulorhexis. The creation of a continuous curvilinear capsulorhexis was found to delay the development of central visual obscuration by facilitating the mechanism of fusion between the edges of the continuous curvilinear capsulorhexis to the posterior capsule, forming a Soemmering's ring. This ring provides a closed environment, which restricts the migration of the LECs toward the central posterior capsule. Incidence of PCO is also high in eyes with uveitis, diabetes, retinitis pigmentation.

- In-the-Bag Fixation. In-the-bag fixation of the optic and the haptic functions primarily to enhance the IOL optic barrier effect, reducing the incidence of central PCO.Tan and Chee noticed an increased incidence of fibrosis-type PCO in cases of ciliary sulcus fixation.

- Anterior Capsule Overlap of IOL Optic. It is believed that with a capsulorhexis smaller than the IOL optic, the adhesion between the anterior capsule and the IOL optic keeps the anterior lens epithelium away from the posterior capsule. This would decrease the incidence of migration of the anterior LECs behind the IOL optic. On the other hand, it has also been postulated that a capsulorhexis larger than the IOL optic allows adhesion of the anterior and posterior capsules, forming a Soemmering's ring. This could contain the cells and regenerative cortical matter, preventing LEC migration onto the visual axis.

- Cortical Cleaving Hydrodissection. The hydraulic force exerted by cortical cleaving hydrodissection causes a cleavage between the lens capsule and the cortex, which could cleave mitotically active LECs from the capsule. High-resolution digital retro illumination images of the posterior capsule in these eyes at 4 years revealed no difference in the incidence of PCO, but the percentage area of the central posterior capsule affected by PCO was significantly lower in eyes that had multiquadrant cortical cleaving hydro dissection than in those that did not. 
- Hydro dissection Combined With Rotation. In an experimental laboratory study of fresh human cadaver eyes, cortical cleaving hydro dissection combined with rotation removed significant quantities of LECs and residual cortical fibres by way of friction.

- Cortical Clean Up. Bimanual irrigation and aspiration for cortical clean up facilitates access to the deep fornices of the capsular bag, especially in the sub incisional quadrants, that have the potential to proliferate and migrate across the central visual axis.

- Bag-in-the-Lens Implantation, The bag-in-the-lens implantation technique has been shown to limit LEC proliferation. In this technique, anterior and posterior capsule flaps of similar sizes are inserted in a flange of the IOL. The LEC proliferation is restricted within the space of the remaining lens bag and does not approach the visual axis. However, it is cautioned that this prevents PCO only if the anterior and posterior capsules have been secured properly in the peripheral groove of the IOL.

- Polishing (Scraping) the Anterior Capsule. Polishing of the anterior capsule has been effective in reducing fibrotic opacification but ineffective in reducing regeneratoryopacification.In eyes with a sharpedged silicone, anterior capsule polishing caused no significant difference in fibrotic PCO.

\section{Iol Design}

- Plate-haptic versus Loop-haptic IOLs. With the plate haptic design IOLs, a high rate of anterior capsule opacification (ACO) as well a high rate of PCO (up to 65\% in standard plate design) has been reported

- Single-piece versus Multipiece IOL Design. No statistical difference has been reported in the rate of development of PCO in the AcrySof three-piece versus the single-piece design.

- Haptic Designs \& Angulation. In addition to the optic edge, it has been observed that the haptic angulation also reduces the incidence of PCO by inducing a pressure gradient over the posterior capsule.

- Accommodating IOL

- $\quad$ Few studies have found increased rates of PCO with the accommodating IOL group postoperatively.

- Biocompatibility. The biocompatibility of IOL materials should be assessed in terms of uveal biocompatibility as well as in terms of capsular biocompatibility. Uveal biocompatibility is related to the inflammatory foreign-body reaction of the eye against the implant. In a study comparing three IOLs: a PMMA IOL, silicone IOL and AcrySof IOL, it was found that all three IOLs were sufficiently biocompatible.

- Bio adhesive IOL Materials. Bioactive materials are those that allow a single LEC to bond both to the IOL and the posterior capsule. This would produce a sandwich pattern including the IOL, the cell monolayer and the posterior capsule. This sealed sandwich structure may prevent further epithelial ingrowth and prevent PCO.Theoretically, a bioactive material such as hydrophobic acrylic would prevent PCO more than PMMA and silicone IOLs, which are biocompatible but also bioinert.

- Various attempts have been made to quantify and grade PCO in the past. Some of the methods used include objective automated quantification of after cataract (AQUA); the subjective evaluation of posterior capsular opacificationsystem (EPCO) and posterior capsule opacificationsoftware (POCO)...

\section{Treatment of pco:}

- Central PCO obscuring the visual axis can be treated with either surgical intervention, such as posterior capsule scraping or with a nonsurgical Nd: YAG laser capsulotomy. The latter method has received wide acceptance. The need for performing capsulotomy depends on the patient's functional impairment of vision, discomfort, demand and the presence of associated risk factors such as high myopia, history of retinal detachment, high risk of cystoids macular edema and only functioning eye. A size that is larger than the pupil diameter under scotopic conditions may prevent disturbances of vision such as monocular diplopia.The clinical complications from Nd: YAG laser capsulotomy include a rise in intraocular pressure, glaucoma, cystoid macular edema and retinal detachment. Nd: YAG laser capsulotomy induces changes in the development and morphology of regeneratory PCO. This response is different with different IOL materials. Silicone and PMMA IOLs led to significant pearl formation on the capsulotomy margin, often combined with a reduction of peripheral regeneratory PCO (silicone). Hydrogel IOLs led to a higher incidence of reclosure of the Nd: YAGcapsulotomy opening. Eyes with acrylic IOLs had no change in regeneratory PCO after the capsulotomy

- Primary posterior capsulotomy allows a clear visual axis, facilitating visual rehabilitation, and reducing the need for Nd.YAGcapsulotomy. The problem in PPCC is the potential closure of the PPCC. Tassignon et al., reported total closure in $6(12 \%)$ of 51 eyes. Vock et al.,found that the visual axis proliferation was significantly lower in PPCC eyes than in nonPPCC eyes. Postoperative retinal complications such as retinal detachment or cystoid macular edema have been shown to be more common with the breakdown of posterior capsule integrity, the lack of aqueous-vitreous barrier disruption after posterior capsulorhexis has 
been previously reported. De Groot et al., showed that breakdown of the ocular barrier occurred only in cases of anterior hyaloid rupture.

Galand et al., reported retinal complications of primary posterior capsulorhexis as a routine procedure during cataract surgery in adults. In their 15 series, 3 of 49 eyes had cystoid macular edema; 3 eyes had vitreous prolapse; and one retinal detachment was noted at postoperative week 4. Lubinski et al., reported that $16 \%$ (4/25) eyes had vitreous prolapse to the anterior chamber.Retinal detachment is a complication of cataract surgery especially in cases that lack capsular integrity. In the general population, the rate of retinal detachment following cataract surgery is $0.6-1.7 \%$. Lubinski et al., reported retinal detachment in only 1 of $25(4 \%)$ patients following cataract surgery with PPCC. However, Nd: YAG laser capsulotomy, which is an alternative procedure to PPCC for treating PCO, is a potential risk factor for retinal detachment.

The incidence of retinal detachment in pseudophakic eyes after YAG capsulotomy has been reported at 1.0-1.9\%.Nd: YAG laser capsulotomy results in uncontrolled posterior capsule disruption that induces posterior vitreous detachment and retinal detachment. In our study, a controlled posterior capsule opening was performed using PCCC without disruption of the anterior hyaloid surface and no retinal detachment was observed in follow-up. We found CDVA was stable from the early postoperative period onward. This outcome was likely due the low rate of PCO and other complications in our study. Additionally, there were no statistically significant differences in mean IOP preoperatively and at all postoperative visits $(\mathrm{P}>0.05$, all cases The IOP outcomes of our study are similar to those reported by Stifter et al., who observed no significant differences in IOP between the cataract surgery with combined PPCC and posterior optic buttonholing and conventional cataract surgery.

\section{Conclusion}

Short term results conclude that cataract surgery with combined PPCC is safe and effective resulting in clinically important advantages such as a low PCO rate, decreased requirement for Nd: YAG laser capsulotomy and long-term prevention of visual morbidity. However a lager randomised study with long follow-up is recommended

\section{References}

[1]. Vasavada AR, Chauhan H, Shah G. Incidence of posterior capsular plaque in cataract surgery. J. Cataract Refract. Surg.23 (5), $798-$ 802 (1997).

[2]. Shirai K, Saika S, Okada Y, Oda S, Ohnishi Y. Histology and immunohistochemistry of fibrous posterior capsule opacification in an infant. J. Cataract Refract. Surg.30 (2), 523-526 (2004).

[3]. Apple DJ, Solomon KD, Tetz MR et al. Posterior capsule opacification. Surv. Ophthalmol.37 (2), 73-116 (1992).

[4]. Rakic JM, Galand A, Vrensen GF. Separation of fibres from the capsule enhances mitotic activity of human lens epithelium. Exp. Eye Res.64 (1), 67-72 (1997).

[5]. Wormstone IM. Posterior capsule opacification: a cell biological perspective. Exp. Eye Res.74 (3), 337-347 (2002).

[6]. Wallentin N, Wickström K, Lundberg C. Effect of cataract surgery on aqueous TGF-beta and lens epithelial cell proliferation. Invest. Ophthalmol. Vis. Sci.39 (8), 1410-1418 (1998).

[7]. Nishi O, Nishi K, Mano C, Ichihara M, Honda T, Saitoh I. Inhibition of migrating lens epithelial cells by blocking the adhesion molecule integrin: a preliminary report. J. Cataract Refract. Surg.23 (6), 860-865 (1997).

[8]. Mathew MR, McLean SM, Murray SB, Bennett HG, Webb LA, Esakowitz L. Expression of CD18, CD49b, CD49c and CD49e on lens anterior capsules in human cataracts. Eye (Lond.) 17(4), 473-477 (2003).

[9]. Volk T, Geiger B. A-CAM: a 135-kD receptor of intercellular adherens junctions. I. Immunoelectron microscopic localization and biochemical studies. J. Cell Biol.103 (4), 1441-1450 (1986).

[10]. Neumayer T, Findl O, Buehl W, Georgopoulos M. Daily changes in the morphology of Elschnig pearls. Am. J. Ophthalmol.141 (3), $517-523$ (2006).

[11]. Kurosaka D, Kato K, Nagamoto T. Presence of $\alpha$ smooth muscle actin in lens epithelial cells of aphakic rabbit eyes. Br. J. Ophthalmol.80 (10), 906-910 (1996).

[12]. Nishi O, Nishi K, Akaishi T, Shirasawa E. Detection of cell adhesion molecules in lens epithelial cells of human cataracts. Invest. Ophthalmol. Vis. Sci.38 (3), 579-585 (1997).

[13]. Schmidbauer JM, Vargas LG, Apple DJ et al. Evaluation of neodymium:yttrium-aluminum-garnetcapsulotomies in eyes implanted with AcrySof intraocular lenses. Ophthalmology 109(8), 1421-1426 (2002).

[14]. Dholakia SA, Vasavada AR. Intraoperative performance and longterm outcome of phacoemulsification in age-related cataract. Indian J. Ophthalmol.52 (4), 311-317 (2004).

[15]. Thompson AM, Sachdev N, Wong T, Riley AF, Grupcheva CN, McGhee CN. The Auckland Cataract Study: 2 year postoperative assessment of aspects of clinical, visual, corneal topographic and satisfaction outcomes. Br. J. Ophthalmol.88 (8), 1042-1048 (2004).

[16]. Schaumberg DA, Dana MR, Christen WG, Glynn RJ. A systematic overview of the incidence of posterior capsule opacification. Ophthalmology 105(7), 1213-1221 (1998)

[17]. Werner L, Pandey SK, Apple DJ. Pathological evaluation of postmortem human eyes implanted with a new single-piece hydrophobic acrylic lens. J. Cataract Refract. Surg.30 (7), 1537-1544 (2004).

[18]. Werner L, Hickman MS, LeBoyer RM, Mamalis N. Experimental evaluation of the Corneal Concept 360 intraocular lens with the Miyake-Apple view. J. Cataract Refract. Surg.31 (6), 1231-1237 (2005).

[19]. Jacob TJ. Human lens epithelial cells in culture: a quantitative evaluation of growth rate and proliferative capacity.Exp. Eye Res.45 (1), 93-104 (1987). 
[20]. Tetz M, Wildeck A. Evaluating and defining the sharpness of intraocular lenses: part 1: influence of optic design on the growth of the lens epithelial cells in vitro. J. Cataract Refract. Surg.31 (11), 2172-2179 (2005).

[21]. Wallentin N, Lundgren B, Holmén JB, Lundberg C. Development of posterior capsule opacification in the rabbit.Ophthalmic Res.34(1), 14-22 (2002).

[22]. Choi J, Park SY, Joo CK. Hepatocyte growth factor induces proliferation of lens epithelial cells through activation of ERK1/2 and JNK/SAPK. Invest. Ophthalmol. Vis. Sci.45 (8), 2696-2704 (2004).

[23]. El-Osta AA, Spalton DJ, Marshall J. In vitro model for the study of human posterior capsule opacification. J. Cataract Refract. Surg.29 (8), 1593-1600 (2003).

[24]. De Jong-Hesse Y, Kampmeier J, Lang GK, Lang GE. Effect of extracellular matrix on proliferation and differentiation of porcine lens epithelial cells. Graefes Arch. Clin. Exp. Ophthalmol.243 (7), 695-700 (2005).

[25]. Mansfield KJ, Cerra A, Chamberlain CG. FGF-2 counteracts loss of TGFbeta affected cells from rat lens explants: implications for PCO (after cataract). Mol. Vis.10, 521-532 (2004).

[26]. McLean SM, Mathew MR, Kelly JB et al. Detection of integrins in human cataract lens epithelial cells and two mammalian lens epithelial cell lines. Br. J. Ophthalmol.89 (11), 1506-1509 (2005).

[27]. Oharazawa H, Ibaraki N, Ohara K, Reddy VN. Inhibitory effects of Arg-Gly-Asp (RGD) peptide on cell attachment and migration in a human lens epithelial cell line. Ophthalmic Res.37 (4), 191-196 (2005).

[28]. Wong TT, Daniels JT, Crowston JG, Khaw PT. MMP inhibition prevents human lens epithelial cell migration and contraction of the lens capsule. Br. J. Ophthalmol.88 (7), 868-872 (2004).

[29]. Ernest PH. Posterior capsule opacification and neodymium:YAGcapsulotomy rates with AcrySof acrylic and PhacoFlex II silicone intraocular lenses. J. Cataract Refract. Surg.29 (8), 1546-1550 (2003).

[30]. Auffarth GU, Brezin A, Caporossi A et al.; European PCO Study Group. Comparison of Nd:YAGcapsulotomy rates following phacoemulsification with implantation of PMMA, silicone, or acrylic intra-ocular lenses in four European countries. Ophthalmic Epidemiol.11 (4), 319-329 (2004). 